\title{
GIS como instrumento catalisador de uma Cidade Inteligente
}

\author{
GIS as a catalyst tool for Smart Cities \\ - Fernando Almeida \\ Universidade Federal de Pernambuco, Brasil \\ f@fernandoalmeida.arq.br \\ - Max Andrade \\ Universidade Federal de Pernambuco, Brasil \\ maxandrade73@gmail.com
}

\begin{abstract}
Every building has its individual and measurable role on resources consumption, waste generation and neighborhood impact within a city, and tracking this behavior is an essential task for establishing a sustainable path into a Smart City model. This paper preliminarily investigates how GIS can contribute in creating an integrated and dynamic system built to attend public utilities and urban management offices for addressing accurate control on saturated and under-risk areas, as well as evaluating sustainability parameters at various scales.
\end{abstract}

Keywords: GIS, Smart Cities, Urban Infrastructure, Public Services, Urban Management

\section{Introdução}

À medida que as cidades têm se tornado ambientes mais complexos, as relações de troca que ali se manifestam tornaramse mais sofisticadas, de modo que em algum momento fez-se necessário estabelecer um conjunto de regras que condicionasse, pelo menos em um primeiro momento, os modos de ocupar e usar o território urbano. Não obstante, ainda que já regrada, a natureza expansiva da cidade provocaria o surgimento de novos desafios, estes subprodutos de uma cada vez mais complexa rede de trocas - redes de mobilidade saturadas, alagamentos, crises de fornecimento de energia e água, manejo ineficiente de resíduos sólidos, decadência das redes físicas urbanas, desequilíbrios microclimáticos, catástrofes naturais, entre outros.

A dinâmica destes eventos dá-se a um passo muito mais célere e intensa que a dinâmica do ferramental da gestão pública, seja este em suas leis ou em seu aparato técnico de capital humano e tecnologias, o que denota a urgência da implementação de mecanismos que permitam monitorar estes pontos críticos detalhada e sistemicamente, e que permitam sua mitigação de forma eficiente.

Em contrapartida a este cenário instável,épossível identificar várias iniciativas ao redor do mundo com o foco, por exemplo, em soluções integradas de tecnologia com a finalidade de promover avanços socioeconômicos nas cidades, e nisto destacam-se os vários conceitos acerca da chamada Smart City, ou Cidade Inteligente (CI). Embora este termo ainda não esteja perfeitamente delineado quanto a uma definição exata, é empregado em diversos países com metodologias e abordagens próprias, mas com a convergência no que se refere ao uso das Tecnologias de Informação e Comunicação (TICS) como base instrumental (HOLLANDS, 2008).

O Sistema de Informação Geográfica (SIG, ou o seu acrônimo em inglês GIS, como é mais difundido) é uma base instrumental já bem estabelecida há décadas nos meios da produção geográfica, cartográfica e de planejamento urbano e regional. Em resumo, trata-se de uma base de dados digital de múltiplas finalidades, na qual um sistema de coordenadas espaciais em comum é o meio básico de referência. Em razão de aglomerar dados oriundos de bases textuais (dados tabulares) a bases gráficas georreferenciadas (mapas, fotos aéreas e afins), consagrou-se como um sistema altamente eficaz para tomada de decisões, ao permitir uma livre manipulação destes dados por meio de pesquisas e combinações variadas, sempre amparadas por uma representação gráfica, vetorial ou raster (FOOTE; LYNCH, 1995).

Emrazãodasuacapacidadedeintegraçãocomosmaisdiversos dados tabulares, o GIS tem se tornado popular no meio da gestão pública por permitir serem elaboradas várias representações gráficas de dados censitários, daí a sua ampla difusão entre órgãos que lidam com indicadores socioeconômicos. Por outro lado, a representação gráfica vetorial dos lotes urbanos em GIS tem sido cada vem mais utilizada para controle do uso e ocupação do solo, mas com foco na arrecadação tributária (RECIFE, 2009).

A partir do princípio de que cada edifício tem sua contribuição individual e mensurável no consumo de recursos (água e energia), geração de resíduos (esgoto e lixo), impacto na vizinhança 
(permeabilidade do solo, retenção de veículos, emissão de ruídos), este artigo busca investigar de modo preliminar como o GIS pode vir a contribuir na criação de um banco de dados integrado às concessionárias públicas e órgãos de gestão urbana para um mapeamento mais preciso das áreas de saturação e risco (aqui, por razões de limitação de escopo, serão abordados o consumo hídrico e energético, e a emissão de esgotos por lote urbanizado, mas seriam também computáveis outros fatores, tais como dimensionamento de tubulações das redes públicas, estrutura de pavimentação, rede de coleta de lixo, efeito térmico da volumetria e materiais dos edifícios, geração de fluxo de entrada e saída de veículos entre outros).

Sistemas de leitura de consumo de energia e água, por exemplo, hoje já são praticados com bases digitais nas capitais brasileiras, o que torna mais viável que estes dados sejam integrados a um banco de dados central e interpretados dentro do GIS. Outras características de desempenho dos edifícios (tais como geração de tráfego potencial em razão do número de vagas de estacionamento, fatores de desempenho térmico das fachadas, emissão de $\mathrm{CO} 2$ da construção, entre outros) podem ser obtidas digitalmente por meio de projetos desenvolvidos em plataforma BIM(Building Information Modeling). Esteúltimo caso, entretanto, é aplicável apenas a uma parcela das novas construções, posto que o BIM ainda é um processo em consolidação no mercado de AEC (arquitetura, engenharia e construção) e raramente empregado nos casos de construções preexistentes (VOLK et al, 2014).

Pressupõe-se, portanto, que qualquer caminho em direção a uma Cidade Inteligente deva ter por base um banco de dados de natureza e robustez tais que permita monitorar e agir de forma mitigadora para lidar com os principais elementos críticos da cidade em prol de um sistema urbano sustentável.

\section{Procedimentos Metodológicos}

Para este estudo toma-se como exemplo o caso da Prefeitura do Recife, que após algumas décadas a trabalhar com uma base cartográfica desatualizada, contratou em 2006 um serviço para construção de uma base de dados contínua, a abranger uma área de cerca de $220 \mathrm{~km} 2$ com fotos digitais aerofotogramétricas de alta definição em escala de 1:6000. A partir de então a Prefeitura deu início a uma mudança de paradigma quanto ao seu modelo operacional, aderindo maciçamente ao uso de GIS e estabelecendo novasrotinasdetrocas deinformaçãoentresecretariasmunicipais, o que permitiu que alguns anos depois fosse implementada na internet uma ferramenta de webGIS de livre acesso público chamada E-SIG (SILVA; NEVES, 2012).

Este sistema, concebido internamente para disseminar informações georreferenciadas da administração municipal, tem como principal funcionalidade servir de base de endereçamento para todos os serviços da Prefeitura. Encontram-se bairros, quadras, lotes, arruamentos e alguns equipamentos públicos, os quais podem ser não apenas visualizados como submetidos a mecanismos de pesquisa (RECIFE, 2010).

Constatou-se, contudo, que esta ferramenta disposta na internet é pouco intuitiva, de modo que a falta de clareza da sua interface não permite transparecer o grau de riqueza dos dados que estão ali vinculados, porém subliminarmente. É apenas por meio do download desta base, também livremente disponível, que é possível manipulá-la em software adequado de GIS e observar os dados tabulares que ali se encontram mas não são facilmente aproveitáveis por meio do E-SIG (ver Figura 1). Por outro lado, vê-se possível criar novos campos tabulares para inserir dados inéditos, desde que compilados de forma criteriosa por meio dos órgãos públicos e concessionárias.

Com os dados existentes, pode-se, por exemplo, valer-se do atributo "QTDPAVIMEN" (nome de atributo arbitrário sob o qual são computados números correspondentes à quantidade de pavimentos das construções de cada lote) para gerar um mapa temáticonoqual sãoatribuídas cores depreenchimentoespecíficas para os lotes em razão de um determinado intervalo de valores (ver Figura 2). Desta forma, pode-se ter uma apreensão bastante clara e precisa, por exemplo, das áreas com as edificações mais altas na cidade. O mesmo pode ser feito a partir do atributo "ANCONSTR" (nome de atributo arbitrário sob o qual são computados números correspondentes ao ano de construção das edificações de cada lote) e gerar um mapa temático que permita identificar as áreas da cidade com os edifícios mais antigos e potencialmente precários.

O que se observa é a capacidade de se incrementar esta base tabular existente dos lotes (na qual constam dados relativos ao gabarito, ao ano de construção, ao número do lote, à área total construída, ao tipo de empreendimento existente, à área do lote e ao comprimento da testada principal) com dados que poderão ser coletados junto às concessionárias de serviços públicos responsáveis pelo fornecimento de água e energia, o que permitiria mapear com idêntica precisão as áreas da cidade com maior consumo energético e hídrico (e neste último caso, também a emissão proporcional de resíduos sanitários).

As informações referentes à previsão do consumo de água, de energia elétrica e emissão de resíduos líquidos sempre fizeram parte da elaboração dos projetos executivos de arquitetura, mesmo nos casos daqueles desenvolvidos à mão. Estas estimativas são caras ao projeto, pois sem os quais não é possível dimensionar os reservatórios, quadros de distribuição e fossas/dutos de escoamento. A partir de um leiaute prévio da ocupação de uma residência, alguns parâmetros são direta ou indiretamente definidos, tais como consumo médio de água por pessoa e por dia (e proporcional despejo de águas cinza e negra), consumo de energia, etc. (SNIS, 2012 apud NINOMYIA et al, 2013).

Esta prática de dimensionamentos no projeto pouco foi afetada após a introdução de ferramentas CAD, exceto pelo aumento da precisão da sua estimativa. Mas estes dados mantemse atrelados a um desenho bidimensional sem "inteligência própria", o que pode ser entendido por um computador apenas como linhas de texto. São, portanto, representados em memoriais descritivos desenvolvidos em processadores de texto, ou em tabelas não integradas, as quais resultam em anexos destes mesmos documentos de texto. Em suma, da prática difundida, mesmo com o uso do CAD, os dados de desempenho do edifício, particularmente os parâmetros de consumo hídrico e energético e emissão de resíduos líquidos, não são aproveitados como dados 


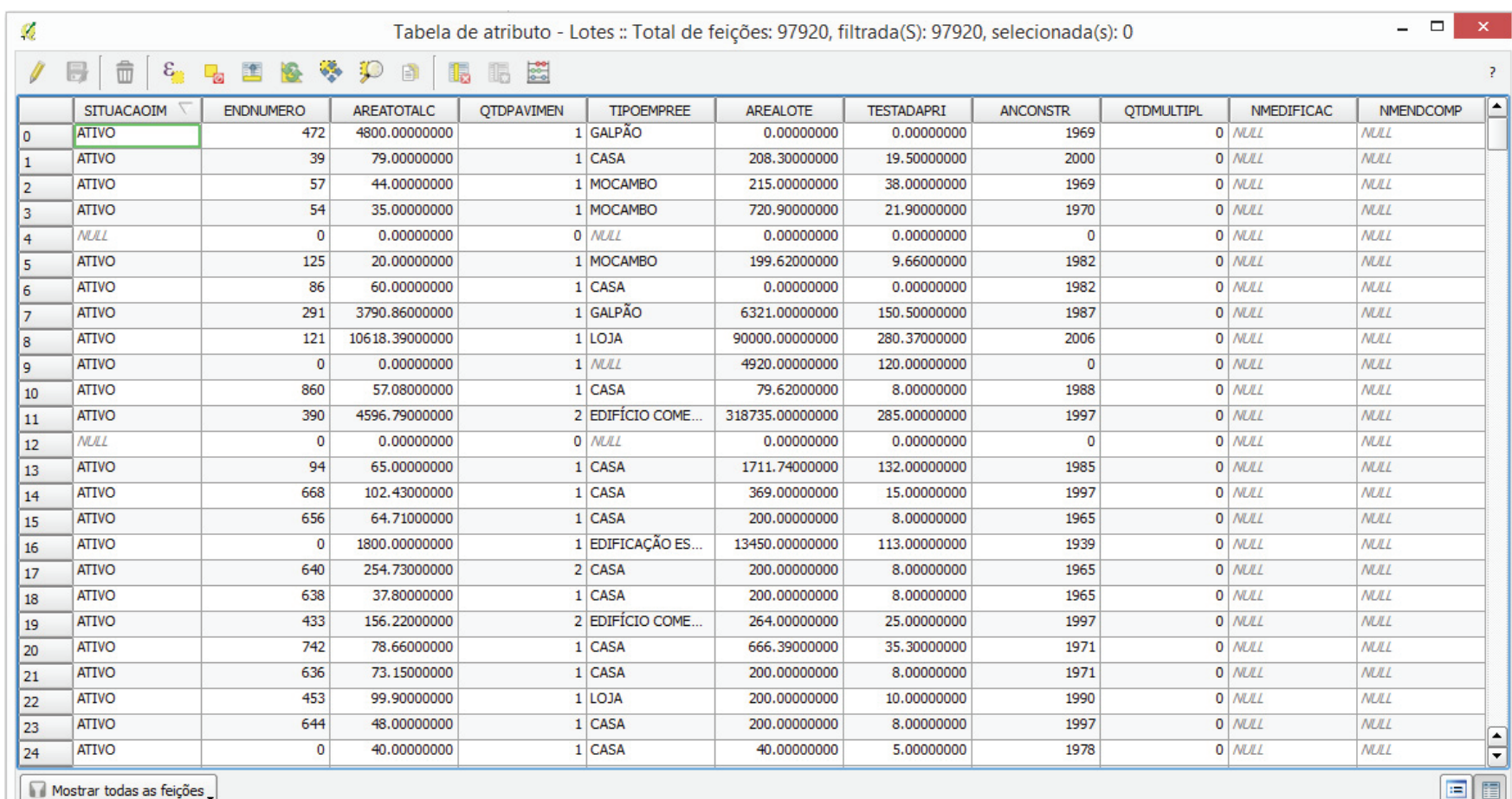

Figura 1: Dados tabulares extraídos do ESIG e expressos em uma tabela de atributos do software GIS.

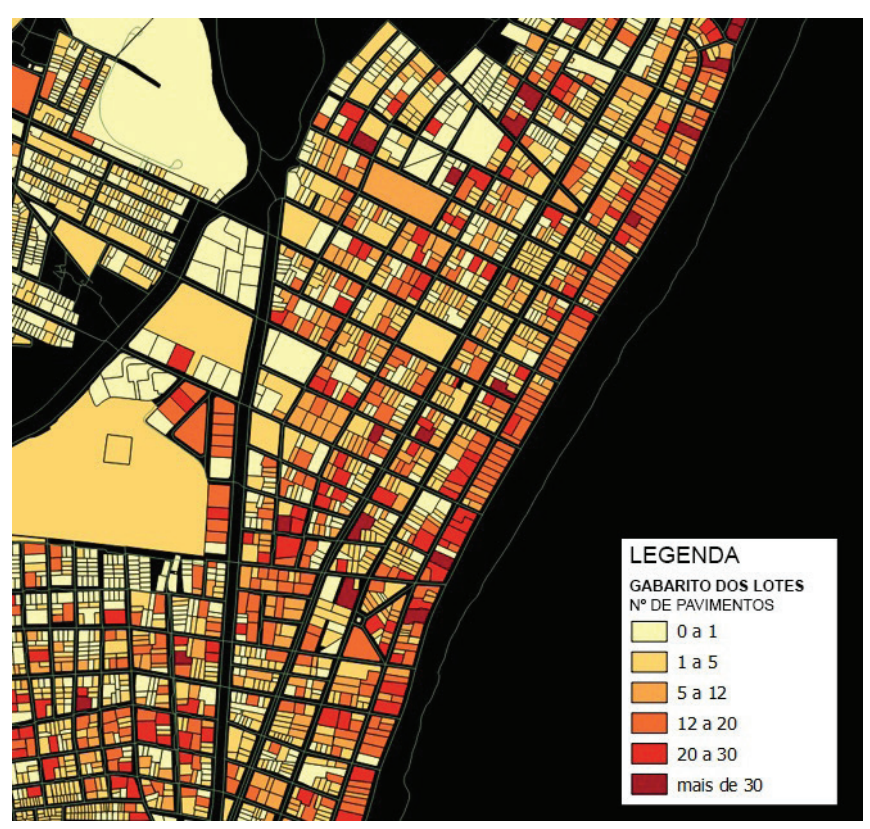

paramétricos digitais.

Figura 2: Exemplo da exibição do atributo QTDPAVIMEN no mapa.

Com a gradativa implementação do BIM, o fundamento de se operar com elementos parametrizáveis e de se poder atribuir dados qualificados a um modelo tridimensional estabelece um novo paradigma na gestão da informação da construção, permitindo que várias ferramentas de análise de desempenho sejam utilizadas com eficiência (ADDOR et al., 2010). Assim, os dados relativos às estimativas de consumo de recursos e emissão de resíduos podem estar integrados ao modelo digital e o controle das informações sobre os impactos da edificação no sítio tende a tornar-se cada vez mais acessível.

O uso do GIS neste contexto é adequado por operar de forma bastante integrável entre distintas interfaces, sejam elas alimentadoras de dados por meio manual (o que se adequa ao modus operandi do projeto arquitetônico tradicional) ou por meio de vínculo a bancos de dados dinâmicos (o que lhe permite comunicar com vários outros sistemas de TI, inclusive o BIM) (NOUR, 2011).

Esta característica marcante do GIS deve-se ao modo como seus arquivos nativos (shapefiles) relacionam-se entre si e com demais fontes. Um shapefile consiste basicamente em um arquivo principal (*.shp), um arquivo-índice (*.shx) e uma tabela dBASE (*.dbf), havendo ainda outros arquivos acessórios que também podem estar associados a um mesmo shapefile. $O$ arquivo principal é um arquivo de acesso direto no qual cada um representa um conjunto de dados de uma mesma natureza de forma (ponto, linha ou polígono) com uma lista de seus vértices. $O$ arquivo-índice tem a finalidade de indexar a leitura do arquivo principal, de modo a permitir que o software faça buscas rápidas no conteúdo deste. A tabela dBASE contém todos os atributos literais atribuídos às feições presentes naquele arquivo principal (FOOTE; LYNCH, 1995; ESRI, 1998).

Com a finalidade de explorar as possíveis aplicações do GIS em relação às informações do conjunto edificado da cidade do Recife, estabeleceu-se um recorte urbano de duas quadras, com um conjunto variado de tipologias construtivas (de casas térreas a torres de 26 andares), em uma área suprida por um amplo conjunto de infraestruturas de serviços urbanos (água, esgoto, energia, gás, TV a cabo, vias pavimentadas, calçadas pavimentadas). Este recorte encontra-se no bairro de Boa 

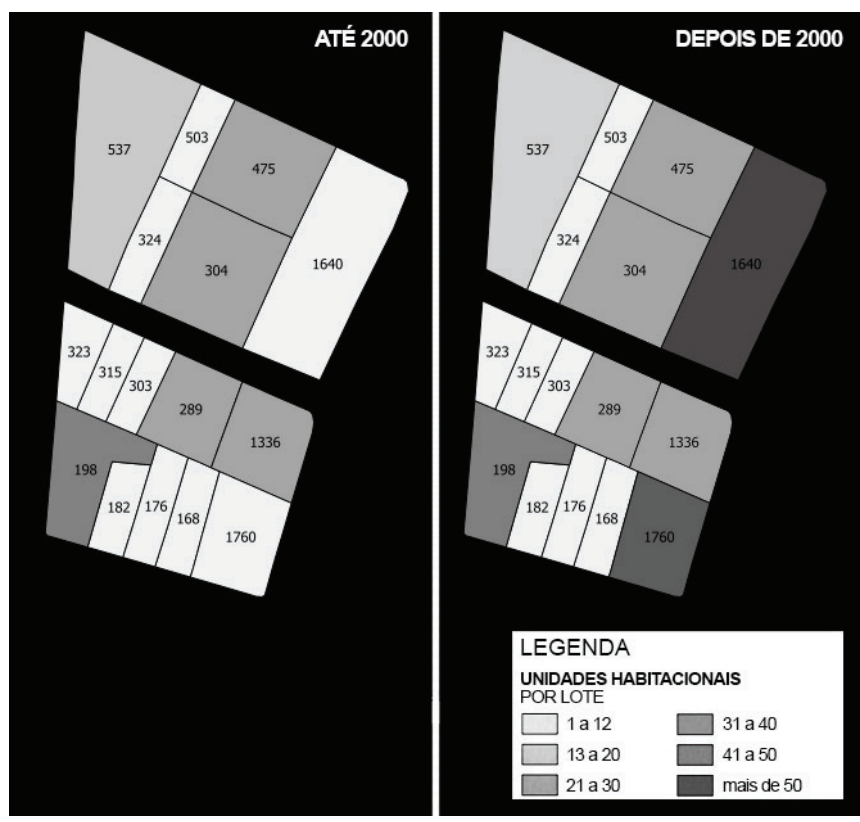

Figura 3: Exemplo da exibição do atributo UNIHAB condicionada ao atributo ANOCONST.

Viagem e consiste em um total atual de 16 lotes. Estes lotes sofreram várias modificações de remembramento ao longo dos anos, entretanto o estudo mantém-se atento às modificações realizadas a partir do ano de 2000.

Nestes últimos 15 anos, dois grandes lotes que antes estavam desocupados deram lugar a um conjunto de tuas torres de 26 andares em um dos lotes (lote 1640), os quais já se encontram em uso, e uma torre de 24 andares no outro, a qual encontra-se em fase final de construção. $O$ que até 2000 somava-se entre as duas quadras um total de 190 unidades habitacionais, em 2016 prevê-se um somatório de 342 unidades, ou seja, um incremento de $80 \%$ na densidade habitacional do entorno.

Na prática, estas informações podem ser incorporadas ao banco de dados dBASE do E-SIG e cruzadas, relacionando os dados contidos no atributo UNIHAB (referente ao número de unidades habitacionais em cada lote, criado pelo autor por meio de inquérito local) com o atributo ANOCONST (ano de construção da edificação). O resultado visual do cruzamento destes dados pode ser visto na Figura 3, onde é apresentado um cenário até o ano 2000 e o outro posterior a este ano, o que permite identificar, além dos lotes com a densidade aumentada, que os mesmos estão situados em uma mesma rua. Este tipo de visualização permite, por exemplo, identificar o potencial de inserção de veículos em determinadas vias, posto que o número de vagas por lote também é um dado conhecido, presente na tabela dBASE como o atributo VAGAS (ver Figura 4).

No que trata do consumo de energia elétrica e água (e geração de resíduos líquidos), embora possa ser estimada uma média de consumo e geração per capita e por classe social, o grau de divergência entre dados estimados e dados


Figura 4: Exemplo da exibição do atributo VAGAS condicionada ao atributo ANOCONST.

reais coletados junto às concessionárias tende a ser elevado. Esta variação dá-se em função da variação de ocupantes por unidade habitacional, de determinados aspectos construtivos de cada unidade (como presença ou não de condicionadores de ar, chuveiros elétricos, bacias sanitárias com válvula de descarga, entre outros equipamentos de elevado consumo) e de hábitos culturais. Por esta razão faz-se necessária a coleta de informações de forma direta junto às concessionárias.

\section{Resultados}

Por estar em fase preliminar, esta pesquisa ainda não estabeleceu o procedimento de levantamento de dados junto às concessionárias. Neste período foi realizada uma revisão bibliográfica e experimentações junto ao software de geoprocessamento, para que fossem indicadas as interseções mais adequadas entre os atributos existentes. Nesta primeira aproximação, os dados médios projetados de consumo serviram para demonstrar as progressões temporais entre os lotes antes e após grandes intervenções de construção.

Por meio desta amostra pretende-se direcionar a elaboração de mapas temáticos dinâmicos e elucidativos de algumas características críticas da ocupação urbana. Com o propósito de extrapolar o uso predominantemente empírico e de caráter censitário dos instrumentos de análise de geoprocessamento, pretende indicar outras informações que também podem ser agregadas à base tabular no intuito de ampliar o escopo do controle urbano. Espera-se que os resultados apresentados confirmem a importância do emprego do GIS para a eficácia do estabelecimento de uma base de dados de análise de desempenho urbano, o que se considera crucial 
mediante qualquer meta de desenvolvimento da cidade. Discussão

Este estudo pretende dar seu contributo na discussão a respeito das bases para o estabelecimento de uma Cidade Inteligente plena, a considerar que quase sempre é uma cidade preexistente que possui esta meta, mesmo que imprecisa. Procura, ainda, levantar questionamentos sobre como lançar as bases para que as TICs que caracterizarão a Cidade Inteligente não sejam eventos isolados, mas uma rede de trocas de informações fundamentada por um banco de dados integrado e consolidado, alimentado de maneira pragmática e gerido de maneira holística.

\section{Referências}

ADDOR, M. R. A. et al. Colocando o "i" no BIM. ARQ.URB. São Paulo: USJT: 104-115 p. 2010.

BITTENCOURT, G. Compesa moderniza sua rede de saneamento. Rio de Janeiro, 2010. Disponível em: < http://www.guiadascidadesdigitais.com.br/site/pagina/ compesa-moderniza-sua-rede-de-saneamento >. Acesso em: 23/02/2015.

ESRI. ESRI Shapefile Technical Description. Redlands, USA, p.1-34.1998.

FOOTE, K. E.; LYNCH, M. Geographic Information Systems as an Integrating Technology: Context, Concepts, and Definitions. Boulder, USA, 1995. Disponível em: < http:// www.colorado.edu/geography/gcraft/notes/intro/intro.html >. Acesso em: 13/01/2015.

HOLLANDS, R. G. Will the real smart city please stand up? City: analysis of urban trends, culture, theory, policy, action. London, UK: Routledge. 12: 303-320 p. 2008.

MCROBERTS, P. How to Design and Build Future Sustainable Cities Using BIM. Guest Column, Fort Collins, EUA, 2010. Disponível em: < http://www.environmentalleader. com/2010/06/25/how-to-design-and-build-futuresustainable-cities-using-bim/ >. Acesso em: 15/03/2015.

NINOMIYA, G. A.; KELLNER, E.; AKUTSU, J. Análise comparativa do consumo de água de dois bairros na cidade de São Carlos (SP). Revista Nacional de Gerenciamento de Cidades. Tupã, SP: Associação Amigos da Natureza da Alta Paulista. 01: 107-122 p. 2013.

NOUR, A. M. The Potential of GIS Tools in Strategic
Urban Planning Process; as an Approach for Sustainable Development in Egypt. Journal of Sustainable Development, v. 4, n. 1, p. 15, 2011. Disponível em: < http://ccsenet.org/ journal/index.php/jsd/article/view/8239/6820 >. Acesso em: 15/03/2015.

OLIVEIRA, P. A. D.; OLIVEIRA, M. P. G. Usos de um Sistema de Informação Geográfica em Cadastro Técnico Municipal: a experiência de Belo Horizonte. Informática Pública. Belo Horizonte: Empresa de Informática e Informação da Prefeitura de Belo Horizonte. 7: 67-84 p. 2005.

ORTH, D.; SILVA, E. Uso da tecnologia GIS em prefeituras brasileiras. III Simpósio Brasileiro de Ciências Geodésicas e Tecnologias da Geoinformação. Recife: UFPE: 1-6 p. 2010.

P., R. R. M.; FAROOQ, J. Integrated infrastructural design and management using BIM and GIS. 9th National GIS Symposium in Saudi Arabia. Damman, Arábia Saudita: SaudiGIS: 1-10 p. 2014.

PERNAMBUCO, G. D. E. D. Sobre o E-SIG. Recife, 2015. Disponível em: < http://www.expressovirtual.pe.gov. br/web/expresso-cidadao-virtual/detalhamento_ item;jsessionid=CD9FE8563D9E26BF57D5170B0FD746CD. server01?p_p_id=publicador_midias_sociais\&p_p_ lifecycle=0\&p_r_p_564233524_itemInteressePK=5517\# >. Acesso em: 13/03/2015.

RECIFE, P. D. C. D. Prefeitura do Recife recebe prêmio internacional. Recife, 2009. Disponível em: < http://www. recife.pe.gov.br/2009/08/24/prefeitura_do_recife_recebe_ premio_internacional_168233.php >. Acesso em: 29/03/2015. SANTOS, S. S. D. Da crise urbana à crise do planejamento urbano: qual papel para os quadros técnicos do planejamento urbano institucionalizado? Encontros Nacionais da ANPUR. Recife: ANPUR. ST2: 18 p. 2013.

SILVA, M. V. D.; NEVES, T. D. F. Mapeamento Digital da Cidade do Recife - PE em SIRGAS 2000. IV Simpósio Brasileiro de Ciências Geodésicas e Tecnologias da Geoinformação. Recife: UFPE: 1-9 p. 2012.

VOLK, R.; STENGEL, J.; SCHULTMANN, F. Building Information Modeling (BIM) for existing buildings - literature review and future needs. Automation in Construction, n. 38, p. 109-127, 2014.

YEH, A. G. O. Urban planning and GIS. 2'. New York: John Wiley \& Sons, 1999.1297. 